Band bending at the heterointerface of GaAs/InAs core/shell nanowires monitored by synchrotron X-ray photoelectron spectroscopy

B. Khanbabaee', G. Bussone, J. V. Knutsson, I. Geijselaers, C. E. Pryor, T. Rieger, N. Demarina, D. Grützmacher, M. I. Lepsa, R. Timm, and U. Pietsch

Citation: J. Appl. Phys. 120, 145703 (2016); doi: 10.1063/1.4964600

View online: http://dx.doi.org/10.1063/1.4964600

View Table of Contents: http://aip.scitation.org/toc/jap/120/14

Published by the American Institute of Physics 


\title{
Band bending at the heterointerface of GaAs/InAs core/shell nanowires monitored by synchrotron X-ray photoelectron spectroscopy
}

\author{
B. Khanbabaee, ${ }^{1, a)}$ G. Bussone, ${ }^{1,2}$ J. V. Knutsson, ${ }^{3}$ I. Geijselaers, ${ }^{3}$ C. E. Pryor, ${ }^{4}$ T. Rieger, ${ }^{5}$ \\ N. Demarina, ${ }^{5}$ D. Grützmacher, ${ }^{5}$ M. I. Lepsa, ${ }^{5}$ R. Timm, ${ }^{3}$ and U. Pietsch ${ }^{1}$ \\ ${ }^{1}$ Solid state physics, Department of Physics, University of Siegen, 57068 Siegen, Germany \\ ${ }^{2}$ Deutsches Elektronen-Synchrotron, Notkestraße 85, 22607 Hamburg, Germany \\ ${ }^{3}$ Nano Lund, Department of Physics, Lund University, 22100 Lund, Sweden \\ ${ }^{4}$ Department of Physics and Astronomy, University of Iowa, Iowa City, Iowa 52242, USA \\ ${ }^{5}$ Peter Grünberg Institute and JARA-FIT, Forschungszentrum Jülich GmbH, 52425 Jülich, Germany
}

(Received 8 July 2016; accepted 27 September 2016; published online 14 October 2016)

\begin{abstract}
Unique electronic properties of semiconductor heterostructured nanowires make them useful for future nano-electronic devices. Here, we present a study of the band bending effect at the heterointerface of GaAs/InAs core/shell nanowires by means of synchrotron based X-ray photoelectron spectroscopy. Different Ga, In, and As core-levels of the nanowire constituents have been monitored prior to and after cleaning from native oxides. The cleaning process mainly affected the Asoxides and was accompanied by an energy shift of the core-level spectra towards lower binding energy, suggesting that the As-oxides turn the nanowire surfaces to n-type. After cleaning, both As and $\mathrm{Ga}$ core-levels revealed an energy shift of about $-0.3 \mathrm{eV}$ for core/shell compared to core reference nanowires. With respect to depth dependence and in agreement with calculated strain distribution and electron quantum confinement, the observed energy shift is interpreted by band bending of core-levels at the heterointerface between the GaAs nanowire core and the InAs shell. Published by AIP Publishing. [http://dx.doi.org/10.1063/1.4964600]
\end{abstract}

\section{INTRODUCTION}

Semiconductor heterostructured nanowires (NWs) are promising candidates for the design of semiconductor junction devices. ${ }^{1}$ In particular, GaAs/InAs core/shell NWs are of interest for a wide range of applications, ${ }^{2}$ since the lower band gap semiconductor InAs with a band gap of $E_{\mathrm{g}, \text { InAs }}$ $=0.35 \mathrm{eV}^{3}$ is grown onto a higher band gap semiconductor GaAs with $\mathrm{E}_{\mathrm{g}, \mathrm{GaAs}}=1.42 \mathrm{eV}^{3}$ forming a type I heterostructure. ${ }^{1}$ Due to the band offset at the interface, a certain number of electrons and holes will diffuse from the wide-gap to the narrow-gap side of the heterostructure, providing an accumulation of electrons in the InAs shell close to the interface. ${ }^{4}$ Moreover, the large lattice mismatch between the core and shell material originates misfit strain which will also modify the band structure at both sides of the interface. Such a band alignment between GaAs and InAs can be used to fabricate, for example, nanoscale tubular conductors, as reported in Ref. 5. The electronic properties of such high-mobility devices can be correlated with structural properties ${ }^{6-8}$ and with the quality of the heterointerface. A better understanding of such correlations requires a detailed analysis of the effective band confinement of these structures, using highly surface- and interfacesensitive techniques.

X-ray photoelectron spectroscopy (XPS) using synchrotron radiation has been known for decades as a powerful tool to investigate the band discontinuities of semiconductor heterojunctions $^{9-11}$ or the quality of surfaces and interfaces buried under thin films of a few nm thickness. ${ }^{12-15}$ Furthermore,

\footnotetext{
a) Author to whom correspondence should be addressed. Electronic mail: khanbabaee@physik.uni-siegen.de
}

this method has been successfully applied for the investigation of the structure and chemical composition of surfaces and interfaces of semiconductors NWs, e.g., to study the composition and removal of surface oxides on InAs NWs ${ }^{16}$ or to probe the size and oxidation dependence of the conductivity in GaAs NWs. ${ }^{17}$

However, there is a lack of XPS studies on core/shell heterostructure NWs, so far. In this work, we report on high resolution XPS investigation of GaAs/InAs core/shell NWs by means of synchrotron radiation, to monitor the band bending effect at the heterointerface and changes in surface electronic states due to the removal of the native surface oxides after a cleaning process.

\section{EXPERIMENTS}

Considering the small inelastic mean free path (IMFP) of probing electrons, the shell thickness is limited to a few $\mathrm{nm}$ in order to probe the heterointerface of the core/shell nanostructures. In particular, we examined core/shell NWs with a $2 \mathrm{~nm}$ thin InAs shell on a GaAs core which was grown by means of self-catalyzed molecular beam epitaxy (MBE) onto a GaAs(111)B substrate covered with $\mathrm{SiO}_{\mathrm{x}}$. The $\mathrm{SiO}_{\mathrm{x}}$ layer was prepared by a thermal spin-coating process from a hydrogen silsesquioxane (HSQ) film. ${ }^{18}$ The GaAs NWs were grown at a substrate temperature of $590^{\circ} \mathrm{C}$ with a Ga rate of $0.075 \mu \mathrm{m}$ $\mathrm{h}^{-1}$ and an $\mathrm{As}_{4}$ beam equivalent pressure of $\sim 1.3 \times 10^{-6}$ mbar for $45 \mathrm{~min} .{ }^{18}$ For the vapor-solid overgrowth of GaAs by InAs, the temperature was reduced to $490^{\circ} \mathrm{C}$ and the Influx was fixed to $0.1 \mu \mathrm{m} \mathrm{h}^{-1}$. The shell growth time was 1 min. For further details on the NWs growth, see Ref. 19. 


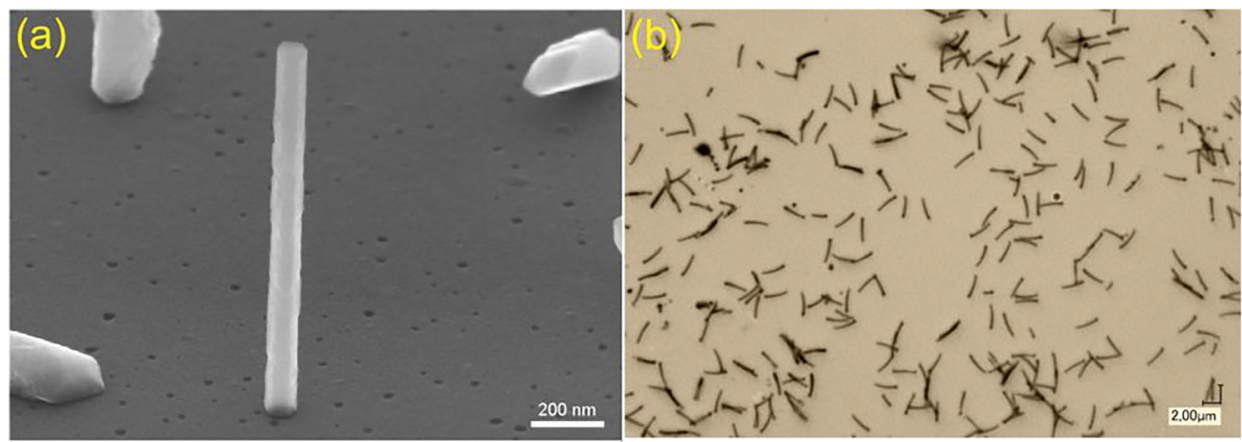

FIG. 1. (a) SEM image of a GaAs/ InAs core/shell nanowire, acquired under an angle of $60^{\circ}$ with respect to the substrate normal. Parasitic growth of crystallites occurs between the nanowires during InAs deposition. (b) A typical optical microscopy image after transferring the nanowires onto a Si wafer.

Fig. 1(a) shows a scanning electron micrograph of a GaAs/InAs core-shell NW. The average length of the GaAs NWs was about $1.5 \mu \mathrm{m}$, and the average core diameter was $(86 \pm 5) \mathrm{nm}$. For XPS measurements, the NWs were harvested from the growth substrate and transferred onto a one-side polished n-doped $\mathrm{Si}$ wafer (see Fig. 1(b)) as described in Ref. 16, to prevent the charging effect and provide a high NW density suitable for inspection with X-ray beams.

The XPS experiments were carried out at beamline I311 of the MAX IV Laboratory synchrotron facility in Lund, Sweden, using a Scienta SES200 electron energy analyzer and a modified plane grating Zeiss SX-700 monochromator. The sample surface was illuminated by X-rays under an incident angle of about $60^{\circ}$, while electrons were collected in the normal direction. As 3d, Ga 2p, Ga 3d, and In 4d core-level spectra were collected at photon energies varying between $170 \mathrm{eV}$ and $1330 \mathrm{eV}$ to allow for compositional depth profiling. ${ }^{20}$ Each spectrum was fitted by one or several doublets (due to spin-orbit splitting) with a Shirley type background and Gaussian (G)-Lorentzian (L) peak functions (Voigt) using the Fityke software. ${ }^{21}$ During fitting, the L-full width at half maximum (FWHM) was kept consistent for all spectral components of the same core level, since it represents the same inherent lifetime broadening. The G-FWHM, however, was kept free during fitting, as it is determined by sample parameters such as crystal quality of the nanowire material, stoichiometric purity of the material (e.g., different suboxides which are difficult to distinguish), but also instrumental broadening. The latter can be as small as $0.04 \mathrm{eV}$ for As $3 \mathrm{~d}$ spectra collected at a photon energy of $170 \mathrm{eV}$ and varies mainly with photon energy of the monochromator and pass energy of the electron analyzer. Details about the used parameters and resulting instrumental broadening can be found in the supplementary material, Table S1. Typical values for the G-FWHM of the bulk (As-Ga) peak in the As $3 \mathrm{~d}$ core-level (Fig. 2(b)) are between 1 and $1.6 \mathrm{eV}$. Our procedure for precisely determining the peak position employs the method of least squares. The binding energies (BEs) of all measured XPS spectra were calibrated to the maximum peak position of $\mathrm{Si} 2 \mathrm{p}$ spectra of the $\mathrm{Si}$ substrate at $98.74 \mathrm{eV}^{20}$

A cleaning process has been performed in the ultrahigh vacuum (UHV) chamber of the XPS setup by exposing the samples to atomic hydrogen at a pressure of $5 \times 10^{-6} \mathrm{mbar}$ and a sample temperature of $400{ }^{\circ} \mathrm{C}$, in order to get rid of the native oxides and additional contamination. Although this procedure was successfully used to remove the native oxides from homogeneous InAs NWs ${ }^{8}$ in the present case, the temperature was too low for a complete removal of native oxides from GaAs NWs. ${ }^{6}$ Unfortunately, a higher temperature could not be used in order to avoid InAs surface degradation and
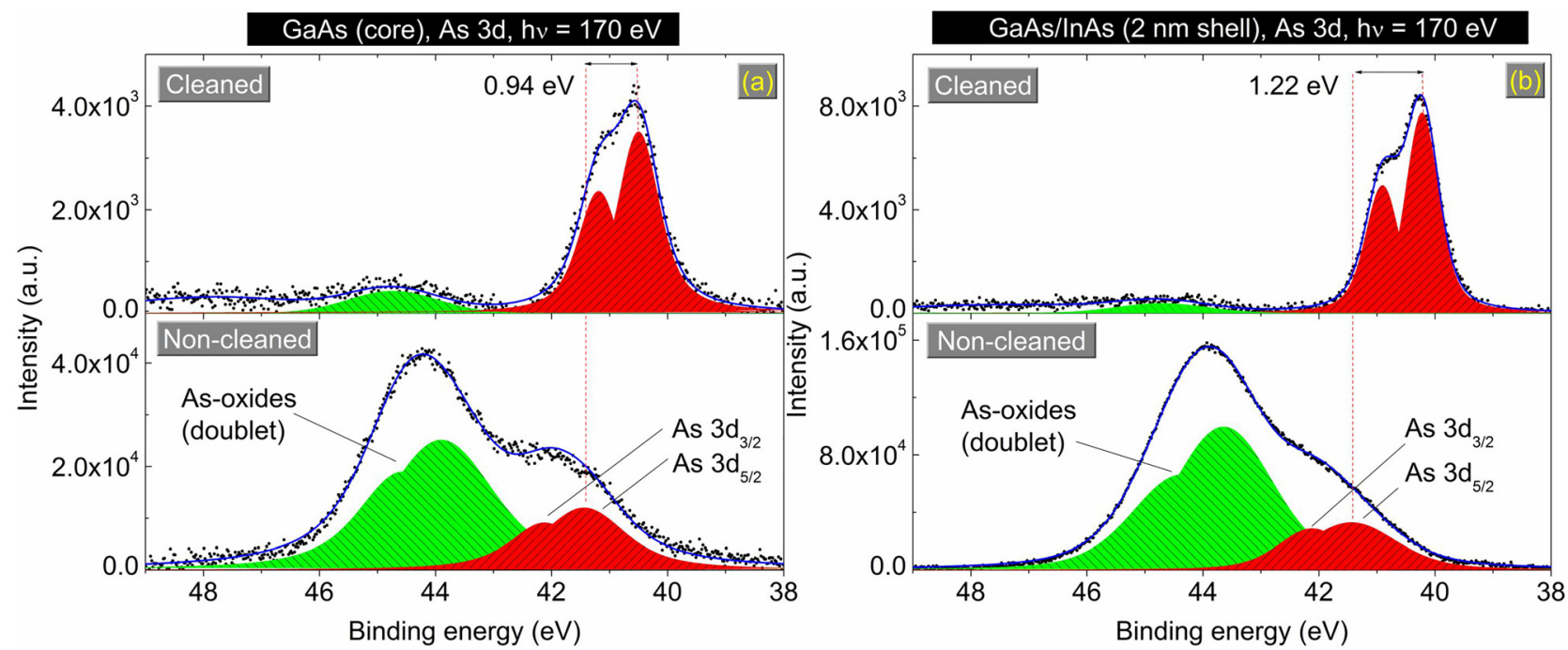

FIG. 2. Comparison of the measured As 3d core-level spectra (dotted curves) of (a) GaAs core and (b) GaAs/InAs core/shell NWs prior to (bottom) and after the cleaning process (top) taken at $\mathrm{h} \nu=170 \mathrm{eV}$. Different fitted components are highlighted by red and green peaks. Blue curves show the sum of the fitted peaks. A Shirley-type background has been subtracted from all data prior to plotting. 
the formation of In droplets. All samples were cleaned by an identical cleaning process.

\section{RESULTS}

\section{A. Cleaning effect}

The measured As 3d core-level spectra of pure GaAs core NWs, collected at the surface sensitive excitation energy of $170 \mathrm{eV}$ prior to (bottom dotted-line curve) and after (top dotted-line curve) cleaning, are illustrated in Fig. 2(a). Each measured profile can be fitted by two doublets: one (red) doublet of each profile is assigned to the As atoms bound to $\mathrm{Ga}$ or In (As-bulk), located at binding energies (BEs) of $41.44 \mathrm{eV}$ and $42.12 \mathrm{eV}$ (prior to cleaning) and BEs of $40.50 \mathrm{eV}$ and $41.18 \mathrm{eV}$ (after cleaning), respectively. Another (green) broad doublet at around 42 to $48 \mathrm{eV}$ can be ascribed to As atoms bound to oxygen (As-oxide). ${ }^{22}$ Because of the large Gaussian width due to multiple sub-oxides, the As-oxide doublet appears only as one large peak after cleaning (Fig. 2(a)-top).

After cleaning, the intensity of this As-oxide peak is drastically decreased, indicating the nearly complete removal of As-oxide from the surface area. This is accompanied by a shift of the As-bulk peak by $0.94 \mathrm{eV}$ towards lower BE. The same cleaning process was carried out for GaAs/InAs core/shell NWs. Fig. 2(b) shows the corresponding measured As 3d spectra prior to (bottom dotted-line curve) and after cleaning (top dotted-line curve). The spin-orbit splitting of As $3 d_{5 / 2}$ and As $3 d_{3 / 2}$ bound to In is located at BEs of $40.22 \mathrm{eV}$ and $40.90 \mathrm{eV}$ (after cleaning), respectively. Also, at this sample, the As-oxide peak (green fitted peak) almost disappeared upon cleaning. Moreover, the decomposition of the measured spectra, using the same fitting process as above, shows an energy shift of about $1.22 \mathrm{eV}$ towards lower BE for As $3 d$ peaks bound to In (red peaks). The origin of these cleaning-induced energy shifts will be discussed later.

\section{B. Shelling effect}

In order to investigate the electronic effect of the InAs shell layer on the As 3d core (shell-effect), the As 3d spectra of the core/shell GaAs/InAs and of the core GaAs NWs after cleaning are compared in Fig. 3(a)-top and -bottom, respectively. It can be seen that the $\mathrm{BE}$ of the As atoms bounded to In at the GaAs/InAs core/shell NWs is about $0.28 \mathrm{eV}$ lower than the BE of the As atoms bounded to Ga at the GaAs core NWs. We examined the observed energy shift for the core/ shell NWs with a nominally thicker shell of $5 \mathrm{~nm}$, as well (not shown). We found a slightly higher energy shift (around $0.35 \mathrm{eV}$ ) for the thicker shell. This observation implies that the origin of this energy shift depends mainly on the shell thickness and is not affected by the charging effect (if any) as reported in Ref. 17.

Furthermore, we investigated the depth dependence of this observed energy shift at the GaAs/InAs heterointerface. We acquired As $3 \mathrm{~d}$ spectra at the higher excitation energy of $350 \mathrm{eV}$ which provides a higher photoelectron kinetic energy of about $300 \mathrm{eV}$, as compared to about $120 \mathrm{eV}$ at an excitation energy of $170 \mathrm{eV}$. This allows us to increase the electron IMFP from $0.6 \mathrm{~nm}$ to $1.0 \mathrm{~nm}$, accordingly. ${ }^{23}$

Fig. 3(b) shows the measured As $3 \mathrm{~d}$ spectra for GaAs/ InAs core/shell NWs after cleaning at $170 \mathrm{eV}$ (top) and $350 \mathrm{eV}$ (bottom). The data of the $350 \mathrm{eV}$ spectrum indeed consist of contributions from both a surface layer component (identical to the spectrum taken at $170 \mathrm{eV}$ ) and components from deeper layers which only become accessible when the photon energy is increased. To highlight this depth profile, we deconvoluted two separate peaks by fitting the $350 \mathrm{eV}$ spectrum using two doublets. We are aware, though, that such a discrete deconvolution is only an approximation for an actually continuous shift in binding energy with increasing probing depth. In the bottom part of Fig. 3(b), the peak energy and FWHM of the red doublet are fixed to the values obtained from the $170 \mathrm{eV}$ fit, while the parameters of the
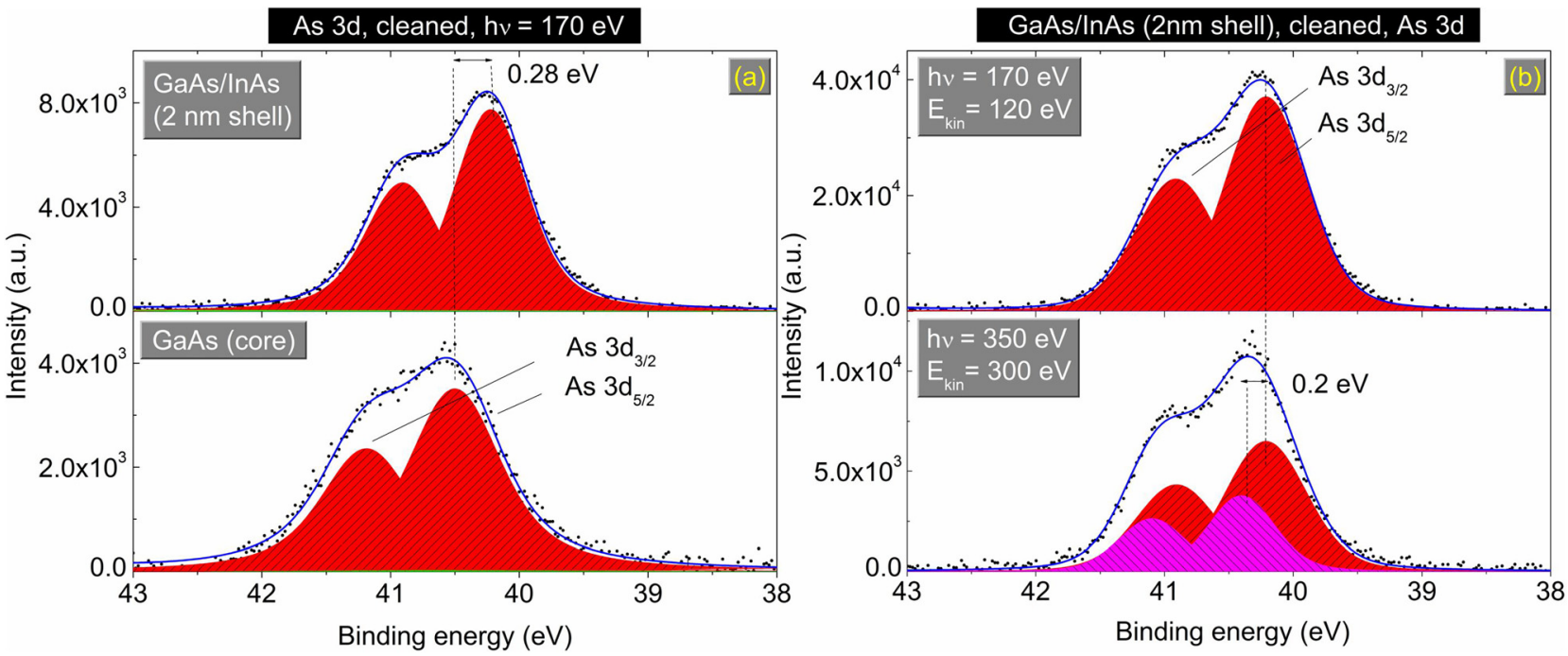

FIG. 3. Comparison of the measured As 3d core-level spectra (dotted curves) of (a) GaAs core (bottom) and GaAs/InAs core/shell NWs (top) after the cleaning process taken at $\mathrm{h} \nu=170 \mathrm{eV}$ and (b) GaAs/InAs core/shell NWs at excitation energies of $\mathrm{h} \nu=170 \mathrm{eV}$ (top) and $\mathrm{h} \nu=350 \mathrm{eV}$ (bottom) after the cleaning process. Different fitted components are highlighted by red and magenta peaks. Blue curves show the sum of the fitted peaks. A Shirley-type background has been subtracted from all data prior to plotting. 
TABLE I. Fit parameters for GaAs/InAs core/shell NWs with $2 \mathrm{~nm}$ thick shell (see Fig. 3(b)).

\begin{tabular}{|c|c|c|c|c|}
\hline \multirow{2}{*}{$\begin{array}{l}\text { Excitation } \\
\text { energy }\end{array}$} & \multicolumn{2}{|c|}{ As $3 d_{5 / 2}$} & \multicolumn{2}{|c|}{ As $3 d_{3 / 2}$} \\
\hline & Red peaks & Magenta peaks & Red peaks & Magenta peaks \\
\hline \multirow[t]{3}{*}{$170 \mathrm{eV}$} & $40.21 \mathrm{eV}$ & & $40.91 \mathrm{eV}$ & \\
\hline & $\mathrm{L}-\mathrm{FWHM}=0.15 \mathrm{eV}$ & & $\mathrm{L}-\mathrm{FWHM}=0.15 \mathrm{eV}$ & \\
\hline & $\mathrm{G}-\mathrm{FWHM}=0.66 \mathrm{eV}$ & & $\mathrm{G}-\mathrm{FWHM}=0.66 \mathrm{eV}$ & \\
\hline \multirow[t]{3}{*}{$350 \mathrm{eV}$} & $40.21 \mathrm{eV}$ & $40.40 \mathrm{eV}$ & $40.91 \mathrm{eV}$ & $41.10 \mathrm{eV}$ \\
\hline & $\mathrm{L}-\mathrm{FWHM}=0.15 \mathrm{eV}$ & $\mathrm{L}-\mathrm{FWHM}=0.15 \mathrm{eV}$ & $\mathrm{L}-\mathrm{FWHM}=0.15 \mathrm{eV}$ & $\mathrm{L}-\mathrm{FWHM}=0.15 \mathrm{eV}$ \\
\hline & $\mathrm{G}-\mathrm{FWHM}=0.66 \mathrm{eV}$ & $\mathrm{G}-\mathrm{FWHM}=0.53 \mathrm{eV}$ & $\mathrm{G}-\mathrm{FWHM}=0.66 \mathrm{eV}$ & $\mathrm{G}-\mathrm{FWHM}=0.53 \mathrm{eV}$ \\
\hline
\end{tabular}

second doublet (shown in magenta) are kept free during fitting. The fitting parameters for GaAs/InAs core/shell NWs are collected in Table I.

By this, we get two distinguishable contributions, one from the surface (red) and another from further within the sample (magenta) at a relative higher BE of $0.2 \mathrm{eV}$. We will come back to these observed energy shifts later.

Here, it should be mentioned that not such a peak broadening with excitation energy was observed for GaAs core NWs (not shown), ruling out again any major influence of the charging effect on the energy shift.

In order to fully investigate the shell-effect at the NW heterointerface, we also wanted to study group-III core-levels; therefore, Ga 2p and Ga 3d core-level spectra have been measured after cleaning. Fig. 4(a) illustrates the measured Ga 2p spectra of GaAs core (bottom) and GaAs/InAs core/ shell NWs (top) taken at an excitation energy of $1330 \mathrm{eV}$, providing a photoelectron kinetic energy of about $200 \mathrm{eV}$ and an electron IMFP of $0.8 \mathrm{~nm}$, which are in the same range as that of the As 3d core-level spectra shown above. These profiles can be decomposed into two doublets: the two red fitted peaks are assigned to the $2 \mathrm{p}_{3 / 2}$ and $2 \mathrm{p}_{1 / 2}$ spin-orbit splitting levels of $\mathrm{Ga}$ bound to As, which were located at around $1116.2 \mathrm{eV}$ and $1143.2 \mathrm{eV}$ for GaAs (core) NWs. The other green fitted doublet can be ascribed to the spin-orbit splitting levels of $\mathrm{Ga}$ bound to oxygen, as, e.g., in $\mathrm{Ga}_{2} \mathrm{O}_{3},{ }^{24}$ which were located at around $1117.4 \mathrm{eV}$ and $1144.4 \mathrm{eV}$ for GaAs (core) NWs. A comparison of the two spectra indicates a shift of about $0.2 \mathrm{eV}$ towards lower BEs for Ga $2 \mathrm{p}$ levels of GaAs/InAs core/shell NWs compared to GaAs core NWs.

Also, Ga 3d and In 4d core-levels, which overlap in BE, have been studied at an excitation energy of $330 \mathrm{eV}$, which corresponds to a photoelectron kinetic energy of about $300 \mathrm{eV}$. The Ga 3d spectra for GaAs core NWs are shown in Fig. 4(b)-bottom. These spectra can be fitted by two doublet peaks. The two red fitted peaks at about $18.7 \mathrm{eV}$ and $19.2 \mathrm{eV}$ are attributed to the $3 \mathrm{~d}_{5 / 2}$ and $3 \mathrm{~d}_{3 / 2}$ levels of Ga bounded to As (Ga-bulk), respectively. A green fitted doublet at around $19.6 \mathrm{eV}$ and $20.1 \mathrm{eV}$ is assigned to Ga-oxides, probably consisting of several sub-oxides. ${ }^{25}$ Furthermore, the tail of a large $\mathrm{O}$ 2s peak from the $\mathrm{SiO}_{\mathrm{x}}$ substrate overlaps in the range of $20-21 \mathrm{eV}$ (cyan). Fig. 4(b)-top shows the corresponding Ga 3d spectra for core/shell NWs. Here, in addition to the two Ga doublets observed for core NWs, four other peaks at around $18.7 \mathrm{eV}, 17.8 \mathrm{eV}$ (a doublet), 17.6 eV, and $16.7 \mathrm{eV}$ (a doublet) can be ascribed to $4 \mathrm{~d}_{3 / 2}$ and $4 \mathrm{~d}_{5 / 2}$ levels of In-oxide (magenta) and In-As (gray), respectively. ${ }^{26}$ A comparison of the fitted Ga-bulk peaks indicates a shift of about $0.25 \mathrm{eV}$ towards lower BE for the Ga $3 \mathrm{~d}$ level of core/shell NWs compared to core NWs. Here, it should be mentioned that in
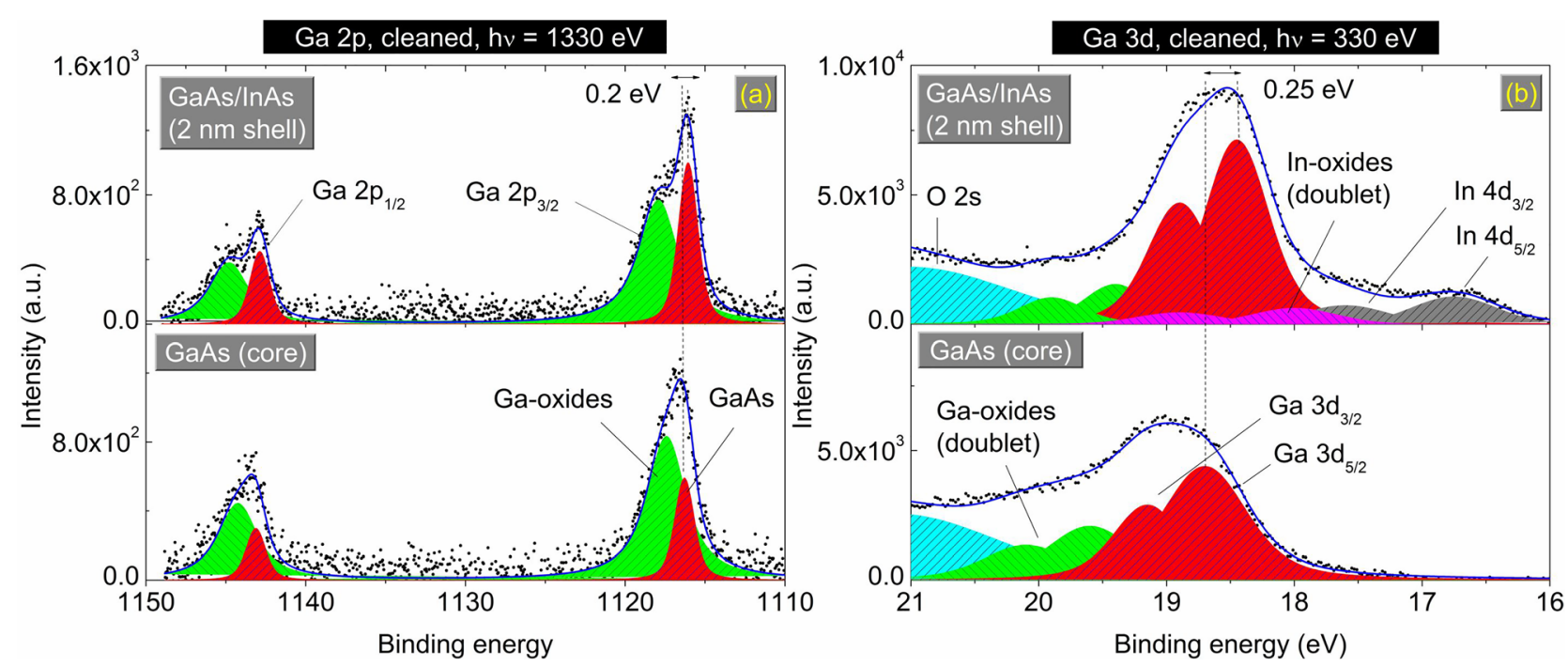

FIG. 4. (a) Comparison of the measured Ga 2p core-level spectra (dotted-line curves) after cleaning, for GaAs core (bottom) and GaAs/InAs core/shell NWs (top) taken at $\mathrm{h} \nu=1330 \mathrm{eV}$. (b) Comparison of the measured Ga 3d-In 4d core-level spectra (dotted-line curves) after cleaning, for the GaAs core (bottom) and GaAs/InAs core/shell (top) taken at $\mathrm{h} \nu=330 \mathrm{eV}$. Different fitted components are highlighted by colored peaks. Blue line curves show the sum of the fitted peaks. A Shirley-type background has been subtracted from all data prior to plotting. 
order to extract the Ga 3d core-level peaks of the core/shell sample, the peak position and FWHM of the O 2s peak and the fitting parameters of the Ga $3 \mathrm{~d}$ peaks, like spin-orbit splitting and branching ratio, were taken similar to those obtained for the GaAs reference sample. By this procedure, we were able to distinguish between the $\mathrm{Ga} 3 \mathrm{~d}$ and In $4 \mathrm{~d}$ components.

Surprisingly, the intensity of the Ga-As component (red doublet) is significantly higher than that of the In-As component (grey doublet) in Fig. 4(b)-top, showing the overlapping $\mathrm{Ga} 3 \mathrm{~d}$ and In $4 \mathrm{~d}$ core-levels. At the excitation energy of $330 \mathrm{eV}$ used here, the XPS cross-section of $\mathrm{Ga} 3 \mathrm{~d}$ is about two times larger than that of In $4 \mathrm{~d}^{27}$ However, with a corresponding electron IMFP of $1.0 \mathrm{~nm}$, the XPS signal should be dominated by photoelectrons coming from the nominally $2 \mathrm{~nm}$ thick InAs shell. From the large Ga signal obtained here, we conclude that a significant amount of Ga atoms may be very close to the NW surface possibly due to strong Ga segregation upon InAs overgrowth, that the InAs layer is thinner than nominally expected, or that the InAs shell is inhomogeneous with some very thin patches that allow a sufficiently high XPS signal from the underlying GaAs core. The latter option is further supported by TEM images of the NW shell, shown in the supplementary material. It should be noted that in XPS spectra from GaAs/InAs NWs with a nominally $5 \mathrm{~nm}$ thick InAs shell (not shown here), the In-As component is significantly larger than the Ga-As component, as expected.

\section{DISCUSSION}

It is well known that the native oxide layers on most III-V semiconductor surfaces pin the Fermi level $\left(\mathrm{E}_{\mathrm{F}}\right)$ close to or in the conduction band. ${ }^{28,29}$ Although, in most cases, the $\mathrm{E}_{\mathrm{F}}$ position was found to remain within the band gap, for some small band-gap semiconductors such as InAs $\mathrm{E}_{\mathrm{F}}$ pinning above the conduction band minimum $(\mathrm{CBM})$ was reported. ${ }^{30-32}$ Considering this, the observed shift of $0.94 \mathrm{eV}$ towards lower $\mathrm{BE}$ upon cleaning for the As $3 \mathrm{~d}$ core-level of GaAs core NWs (see Fig. 2(a)) can be attributed to the un-pinning of the $E_{F}$ due to the removal of native oxide. This is in the range of values reported for bulk GaAs which are between 0.8 and $1.4 \mathrm{eV}$, depending on oxygen exposure. ${ }^{28}$ Furthermore, while with the presently applied cleaning process, the Ga- and In-components of the native oxides partially remained on the NWs surface (see Figs. 4(a) and 4(b)), the As-component was almost completely removed (see Figs. 2(a) and 2(b)). Thus, one can conclude that the observed shift towards lower binding energy is mainly due to the removal of the As-component of the native oxides and that As-oxides are the dominant factor for the strongly n-type character of uncleaned GaAs and InAs NW surfaces.

A similar shift was also observed for GaAs/InAs core/ shell NWs upon cleaning, but with a slightly larger amount of $1.2 \mathrm{eV}$ (see Fig. 2(b)), confirming the dominance of the As-oxides on the surface. It can also not be excluded that weak charging of the oxidized and slightly inhomogeneous NW shell (as shown by TEM $^{33}$ images, see supplementary material) occurs during the XPS measurements, partly contributing to the observed rather large energy shift.

Before we can come to a detailed analysis of the shell effect, meaning band-bending effects at the GaAs/InAs heterointerface as observed by BE shifts in the corresponding core-level spectra, we have to consider possible quantum-size confinement effects in the very thin InAs shell layer. Since the shell thickness of $2 \mathrm{~nm}$ is far below the de Broglie wavelength of electrons in InAs, one should expect a confinement of the electronic states along the radial direction. Such confinement effects have been experimentally observed both for thin InAs layers on GaAs substrates ${ }^{34}$ and for GaAs/InAs core/shell NWs. ${ }^{5}$ In addition, the band gap of the InAs shell as well as that of the GaAs core close to the heterointerface will be significantly influenced by strain due to the large lattice misfit between the two materials. ${ }^{35,36}$

We calculated the strain distribution of our GaAs/InAs core/shell NWs, the resulting band alignment around the heterointerface, and the confinement energy for electrons in the InAs shell in terms of linear elasticity theory. Since the shell layer is $2 \mathrm{~nm}$ thick and the NW core has a diameter of $86 \mathrm{~nm}$, we could not do a full 3D modelling due to limitations in computer power. Instead, we simulated the structure as a quantum well (shell layer) on a [100]-oriented substrate using a $150 \times 150 \times 50$ grid. This approximation will introduce a very small error, less than that caused by the uncertainty in the actual geometry of the NW. We first obtained the strain tensor elements using linear elasticity theory which gave the energy of the band edges. The confinement energy was then computed using a one band model where the vacuum was simulated by a $5 \mathrm{eV}$ barrier. A more detailed description of our method is given in Ref. 37.

The results are plotted in Fig. 5(a): Due to the tensile strain, the size of the GaAs band gap decreases slightly towards the interface, while the band gap of the compressively strained InAs shell amounts to $0.5 \mathrm{eV}$, which is larger than in bulk InAs. In addition to the influence of the strain, we also considered the influence of quantum confinement and calculated the resulting energy of the electron ground state in the InAs shell, which is $0.39 \mathrm{eV}$ above the CBM. Nevertheless, this energy is still $0.14 \mathrm{eV}$ below the CBM of the GaAs, meaning that the confined electron states of the InAs shell lie within the GaAs band gap. This result agrees well with our experimental observation, as will be explained in the following paragraph. It should be noted that the calculations assume an abrupt material interface and a homogeneously $2 \mathrm{~nm}$ thin InAs shell layer. Segregation effects at the $\mathrm{GaAs} / \mathrm{InAs}$ interface and inhomogeneities of the shell thickness are expected to reduce the confinement energy in the InAs shell and move the energy of the electron ground state further away from the GaAs conduction band.

The conduction band offset at a heterointerface between two semiconductors is determined by the electron affinities of the two materials, as shown schematically in Fig. 5(b). Additionally, in equilibrium conditions, the Fermi levels of both semiconductors have to coincide at the interface, which often results in local band bending in the interface region. Such a band bending can lead to transfer of charge carriers across the interface and the formation of space charge layers. The vacuum level follows the slope of the conduction bands, resulting here in a band bending $\Delta \mathrm{E}_{\mathrm{vac}}=\Delta \mathrm{E}_{\mathrm{GaAs}}+\Delta \mathrm{E}_{\mathrm{InAs}}$ (see Fig. 5(b)). Also, the core-levels of the NW atoms follow this slope, since their binding energies are coupled to the 


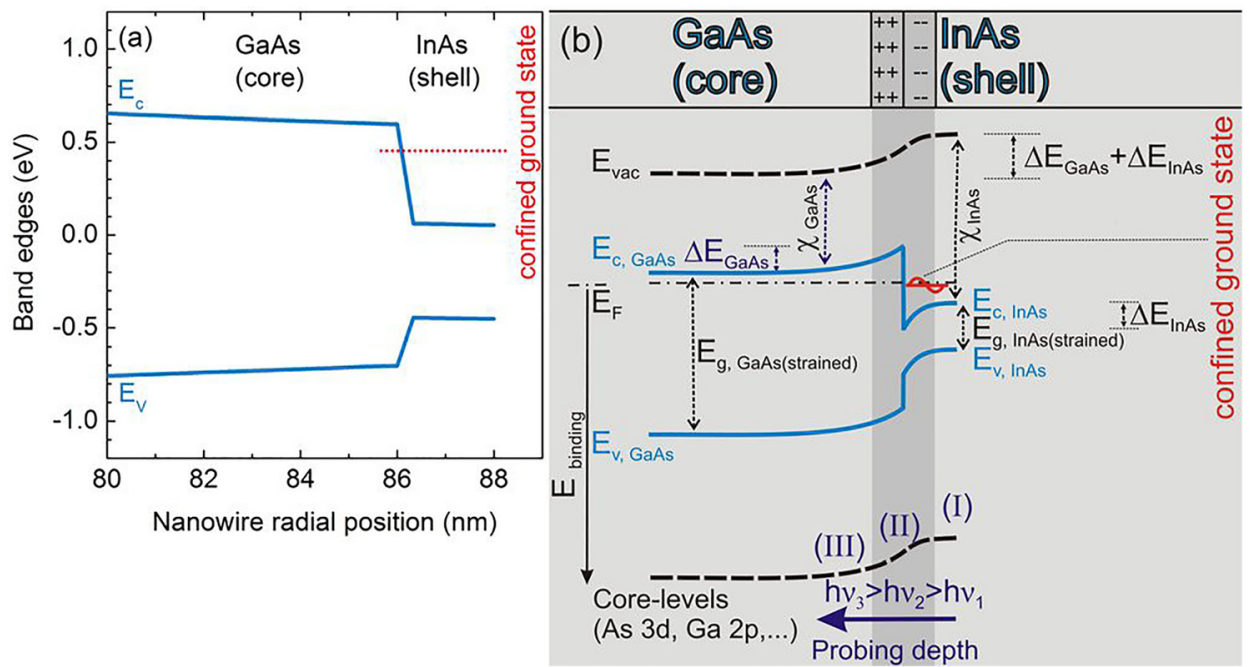

FIG. 5. (a) Calculated alignment of conduction $\left(\mathrm{E}_{\mathrm{C}}\right)$ and valence $\left(\mathrm{E}_{\mathrm{V}}\right)$ band edges (blue lines) around the GaAs/InAs core/shell heterointerface, considering strain due to the NW geometry, but neglecting any band-bending effects. In addition to strain, also quantum confinement is considered for the electron ground state in the thin InAs shell, as indicated by red dots. (b) Qualitative diagram of the band alignment including band-bending effects at the core/shell heterointerface: Adjustment of the Fermi level in the GaAs core and the filled electron ground state in the InAs shell results in the transfer of electrons from the GaAs side to the InAs side of the interface and the formation of positive (+) and negative (-) space charge layers. The corresponding band bending, amounting to $\Delta \mathrm{E}_{\mathrm{GaAs}}+\Delta \mathrm{E}_{\mathrm{InA}}$, affects the conduction and valence bands as well as the vacuum level and the core-levels. The probing depth through the InAs shell layer (I) towards the GaAs core NW (III) over the heterointerface (II) can be realized by increasing the excitation energies from $\mathrm{h} \nu_{1}$ to $\mathrm{h} \nu_{3}$ over $\mathrm{h} \nu_{2}$. Energy levels and distances are not drawn to scale.

vacuum level. In contrast, the underlying Si substrate and its Fermi level are not affected by the deposited NWs, in particular, by the cleaning procedure performed here (as much higher temperature is needed to modify Si surface composition). Thus, by monitoring the changes in $\mathrm{BE}$ of the $\mathrm{NW}$ core-levels relative to the Si Fermi level, we can assess the bending of the NW conduction band. In the present case, such evaluation can be made by comparing the measured core-levels of the core/shell NWs with those of reference core NWs, where no interface band bending occurs. Since XPS is a very surface-sensitive technique, the XPS signal from the core/shell NWs is dominated by electrons that have been emitted from the InAs shell, even though the shell thickness is only $2 \mathrm{~nm}$. For all investigated As $3 \mathrm{~d}$, Ga 3d, and Ga $2 p$ core-levels, the measured binding energies of the cleaned GaAs/InAs core/shell NWs are 0.2 to $0.3 \mathrm{eV}$ smaller than the corresponding values of the GaAs NWs, as shown in Figs. 3(a), 4(a), and 4(b). This means that the probed corelevels of the NW shell are closer to the Fermi energy than those of the NW core, implying that the GaAs bands bend upward and the InAs bands bend downward at the core/shell interface, as shown in Fig. 5(b). Thus, we expect an accumulation of electrons in the InAs shell close to the core/shell interface, similar to the two-dimensional electron gas that has previously been observed at the surface of bulk InAs. ${ }^{38}$ In the present case of a very thin InAs shell, the electrons are confined in discrete energy states instead of a continuous conduction band. But, if we assume a slightly or moderately n-type GaAs core, the ground state and first excited states of the InAs shell are still located below the Fermi level, resulting in electron accumulation in the InAs and the formation of a space charge region across the interface.

The entire amount of band bending across the heterointerface, $\Delta \mathrm{E}_{\mathrm{vac}}=\Delta \mathrm{E}_{\mathrm{GaAs}}+\Delta \mathrm{E}_{\mathrm{InAs}}$, is obtained when comparing
XPS spectra acquired at the surface region of core/shell NWs with those acquired at the reference core NWs. This is the case for As 3d spectra taken at a low photon energy of $170 \mathrm{eV}$ if we assume the same binding energy for As-Ga and As-In. ${ }^{25}$ According to Fig. 3(a), we thus obtain the amount of $0.28 \pm 0.04 \mathrm{eV}$ for the total band bending $\Delta \mathrm{E}_{\mathrm{GaAs}}$ $+\Delta \mathrm{E}_{\text {InAs. }}$. In addition, it is possible to map the band bending by monitoring the $\mathrm{BE}$ of a core-level as a function of probing depth through the InAs shell layer towards the heterointerface of core/shell NWs or even beyond. This can be realized by increasing the electron IMFP, i.e., by increasing the photoelectron kinetic energy with appropriate excitation energies, as indicated in Fig. 5(b) by regions (I), (II), and (III). A comparison of As 3d spectra obtained from the GaAs/InAs core/shell NWs at photoelectron kinetic energies of $120 \mathrm{eV}$ and $300 \mathrm{eV}$ is shown in Fig. 3(b). At a kinetic energy of $120 \mathrm{eV}$, corresponding to an electron IMFP of $0.6 \mathrm{~nm}$, the majority of detected photoelectrons originates from the NW surface (red doublet in Fig. 3(b)). At $300 \mathrm{eV}$, corresponding to an IMFP of $1.0 \mathrm{~nm}$, one should expect that photoelectrons from the interface region are detected in addition to the surface electrons, and the resulting XPS spectrum becomes a combination of both contributions. Since we have seen above that the InAs shell is probably inhomogeneous with some areas being significantly thinner than the nominal $2 \mathrm{~nm}$, we can expect a substantial contribution from the interface region in the $300 \mathrm{eV}$ spectra. Indeed, the $300 \mathrm{eV}$ spectrum in Fig. 3(b) shows a combination of both contributions, which can be distinguished by fitting a surface-related doublet (red peaks) and an interface-related doublet (magenta peaks), which are separated in $\mathrm{BE}$ by $0.19 \mathrm{eV}$. According to Fig. 5(b), this BE shift between surface and interface contributions can roughly be considered as the amount of band bending within the InAs shell, $\Delta \mathrm{E}_{\mathrm{InAs}}$. 
A suitable way to evaluate the band bending within the GaAs core at the interface, $\Delta \mathrm{E}_{\mathrm{GaAs}}$, is to compare $\mathrm{Ga} 2 \mathrm{p}$ or $\mathrm{Ga} 3 \mathrm{~d}$ core-level spectra acquired at GaAs/InAs core/shell NWs with those acquired at reference core NWs, since the corresponding photoelectrons should always originate from the GaAs material. The measured shifts in $\mathrm{BE}$ are $0.2 \mathrm{eV}$ for Ga 2p (Fig. 4(a)) and $0.25 \mathrm{eV}$ for Ga 3d (Fig. 4(b)). However, it cannot be excluded that some Ga material has segregated into the InAs shell, as discussed above. In this case, the observed shift in BE would reflect both the band bending in the GaAs core and at least partially the band bending in the InAs shell. Considering the slightly inhomogeneous thickness of the InAs shell layer (as can be seen from the TEM images shown in the supplementary material), and taking into account the shift in BE between surface and interface contributions in the As 3d spectra discussed above, our data from various XPS core-levels result in a congruent picture of the core/shell band bending: We obtain a total band bending of $0.28 \pm 0.04 \mathrm{eV}$, which we estimate to consist of a component in the InAs shell, $\Delta \mathrm{E}_{\text {InAs }}$, of between $0.15 \mathrm{eV}$ and $0.20 \mathrm{eV}$, and a component in the GaAs core, $\Delta \mathrm{E}_{\mathrm{GaAs}}$, of between $0.10 \mathrm{eV}$ and $0.15 \mathrm{eV}$. It should be noticed that the observed shift in BE within the GaAs core can also partly be explained by the strain-induced decrease of the GaAs band gap close to the core/shell interface, as shown in Fig. 5(a). However, this strain-induced shift of the GaAs valence band edge is calculated to be less than $0.02 \mathrm{eV}$ within the outermost $2 \mathrm{~nm}$ of the GaAs core, and therefore of minor relevance compared to the observed bandbending.

\section{CONCLUSION}

In summary, we have studied the heterointerface of GaAs/InAs core/shell NWs using synchrotron radiation XPS. Different core-levels were examined to test and determine the band bending at the heterointerface. Our results suggest that the shelling of the GaAs NWs with $2 \mathrm{~nm}$ InAs leads to band bending of about $0.3 \mathrm{eV}$ at the heterointerface. This is consistent with interfacial strain and quantum confinement in the thin InAs shell layer, as calculated based on elasticity theory, resulting in an electron ground state energy still below the GaAs conduction band edge. Furthermore, while the cleaning process removed only partially the In- and Gaoxides from the NW surface, the As-oxide component of the native oxide was almost completely removed, resulting in a shift of the BE of about 0.9 to $1.2 \mathrm{eV}$ towards lower energies. This result suggests that the As component of the native oxide turns the NW surfaces strongly n-type. While the GaAs/InAs core/shell NW system was chosen here due to its technological relevance, the applied method is not restricted to a specific material system. In contrast, our results reveal the large potential of synchrotron-based XPS studies for investigating detailed electronic properties of hidden semiconductor nanostructure interfaces.

\section{ACKNOWLEDGMENTS}

This work was partially supported by DFG (Pi 217/38). Parts of this work were performed within the NanoLund
Center for Nanoscience at Lund University and were supported by the Swedish Research Council (VR) and the Knut and Alice Wallenberg Foundation. The authors would like to thank M. Hjort (Lund University, now Stanford University) for XPS sample preparation, J. Osiecki and $\mathrm{K}$. Thanell for their support at beamline I311 of the MAX IV Laboratory, and M.-E. Pistol (Lund University) for support with the calculations.

${ }^{1} \mathrm{P}$. Harrison, Quantum Wells, Wires and Dots: Theoretical and Computational Physics of Semiconductor Nanostructures (John Wiley and Sons, 2005).

${ }^{2}$ Ö. Gül, N. Demarina, C. Blömers, T. Rieger, H. Lüth, M. Lepsa, D. Grützmacher, and T. Schäpers, Phys. Rev. B 89, 045417 (2014).

${ }^{3}$ I. Vurgaftman, J. Meyer, and L. Ram-Mohan, J. Appl. Phys. 89, 5815 (2001).

${ }^{4}$ C. Beenakker and H. van Houten, Solid State Phys. 44, 1-28 (1991).

${ }^{5}$ C. Blömers, T. Rieger, P. Zellekens, F. Haas, M. Lepsa, H. Hardtdegen, Ö. Gül, N. Demarina, D. Grützmacher, and H. Lüth, Nanotechnology 24, 035203 (2013).

${ }^{6}$ M. Hjort, S. Lehmann, J. Knutsson, R. Timm, D. Jacobsson, E. Lundgren, K. Dick, and A. Mikkelsen, Nano Lett. 13, 4492 (2013).

${ }^{7}$ G. Bussone, H. Schäpers-Eberwein, E. Dimakis, A. Biermanns, D. Carbone, A. Tahraoui, L. Geelhaar, P. Haring Bolívar, T. U. Schuülli, and U. Pietsch, Nano Lett. 15, 981 (2015).

${ }^{8}$ M. Hjort, S. Lehmann, J. Knutsson, A. A. Zakharov, Y. A. Du, S. Sakong, R. Timm, G. Nylund, E. Lundgren, and P. Kratzer, ACS Nano 8, 12346 (2014).

${ }^{9}$ C. Ke, W. Zhu, Z. Zhang, E. S. Tok, and J. Pan, Surf. Interface Anal. 47, 824 (2015).

${ }^{10}$ G. Ye, H. Wang, and R. Ji, Appl. Phys. Express 8, 081002 (2015).

${ }^{11}$ H. Sato, M. R. Sarkar, Y. Naoi, and S. Sakai, Solid-State Electron. 41, 205 (1997).

${ }^{12}$ B. Khanbabaee, D. Lützenkirchen-Hecht, R. Hübner, J. Grenzer, S. Facsko, and U. Pietsch, J. Appl. Phys. 116, 024301 (2014).

${ }^{13}$ R. Timm, A. Fian, M. Hjort, C. Thelander, E. Lind, J. N. Andersen, L.-E. Wernersson, and A. Mikkelsen, Appl. Phys. Lett. 97, 132904 (2010).

${ }^{14}$ G. He, X. Chen, and Z. Sun, Surf. Sci. Rep. 68, 68 (2013).

${ }^{15}$ C. Hinkle, E. Vogel, P. D. Ye, and R. Wallace, Curr. Opin. Solid State Mater. Sci. 15, 188 (2011).

${ }^{16}$ R. Timm, M. Hjort, A. Fian, B. Borg, C. Thelander, J. N. Andersen, L.-E. Wernersson, and A. Mikkelsen, Appl. Phys. Lett. 99, 222907 (2011).

${ }^{17}$ F. Jabeen, S. Rubini, F. Martelli, A. Franciosi, A. Kolmakov, L. Gregoratti, M. Amati, A. Barinov, A. Goldoni, and M. Kiskinova, Nano Res. 3, 706 (2010).

${ }^{18}$ T. Rieger, S. Heiderich, S. Lenk, M. I. Lepsa, and D. Grützmacher, J. Cryst. Growth 353, 39 (2012).

${ }^{19}$ T. Rieger, M. Luysberg, T. Schäpers, D. Grützmacher, and M. I. Lepsa, Nano Lett. 12, 5559 (2012).

${ }^{20}$ R. Timm, M. Hjort, A. Fian, C. Thelander, E. Lind, J. N. Andersen, L.-E. Wernersson, and A. Mikkelsen, Microelectron. Eng. 88, 1091 (2011).

${ }^{21}$ M. Wojdyr, J. Appl. Crystallogr. 43, 1126 (2010).

${ }^{22}$ S. Ingrey, W. Lau, and R. Sodhi, J. Vacuum Sci. Technol. A 7, 1554 (1989).

${ }^{23}$ S. Tanuma, T. Shiratori, T. Kimura, K. Goto, S. Ichimura, and C. Powell, Surf. Interface Anal. 37, 833 (2005).

${ }^{24}$ M. Milojevic, C. Hinkle, F. Aguirre-Tostado, H. Kim, E. Vogel, J. Kim, and R. Wallace, Appl. Phys. Lett. 93, 252905 (2008).

${ }^{25}$ B. Brennan and G. Hughes, J. Appl. Phys. 108, 053516 (2010).

${ }^{26}$ M. Kobayashi, P. Chen, Y. Sun, N. Goel, P. Majhi, M. Garner, W. Tsai, P. Pianetta, and Y. Nishi, Appl. Phys. Lett. 93, 182103 (2008).

${ }^{27}$ J. Yeh and I. Lindau, At. Data Nucl. Data Tables 32, 1 (1985).

${ }^{28}$ W. Spicer, I. Lindau, P. Skeath, and C. Su, J. Vacuum Sci. Technol. 17, 1019 (1980).

${ }^{29}$ E. Yablonovitch, B. Skromme, R. Bhat, J. Harbison, and T. Gmitter, Appl. Phys. Lett. 54, 555 (1989).

${ }^{30}$ V. Y. Aristov, G. Le Lay, P. Soukiassian, K. Hricovini, J. Bonnet, J. Osvald, and O. Olsson, EPL (Europhys. Lett.) 26, 359 (1994).

${ }^{31}$ S. Bhargava, H.-R. Blank, V. Narayanamurti, and H. Kroemer, Appl. Phys. Lett. 70, 759 (1997). 
${ }^{32}$ H. U. Baier, L. Koenders, and W. Mönch, J. Vacuum Sci. Technol. B 4, 1095 (1986).

${ }^{33}$ A. Thust, J. Barthel, and K. Tillmann, J. Large-Scale Res. Facil. 2, A41 (2016).

${ }^{34}$ H. Yamaguchi, J. Sudijono, B. Joyce, T. Jones, C. Gatzke, and R. Stradling, Phys. Rev. B 58, R4219 (1998).
${ }^{35}$ J. Arbiol, M. De La Mata, M. Eickhoff, and A. F. i Morral, Mater. Today 16, 213 (2013).

${ }^{36}$ S. Raychaudhuri and E. Yu, J. Appl. Phys. 99, 114308 (2006).

${ }^{37}$ C. Pryor, Phys. Rev. B 57, 7190 (1998).

${ }^{38} \mathrm{H}$. Lüth, Surfaces and Interfaces of Solids (Springer-Verlag, Berlin, Heidelberg, 1993). 